\title{
Drug interactions with oral hypoglycaemic drugs
}

\author{
Gillian M. Shenfield, Professor in Clinical Pharmacology, Department of \\ Clinical Pharmacology, Royal North Shore Hospital, Sydney
}

\section{SYNOPSIS}

Oral hypoglycaemic drugs may interact with other drugs. Pharmacodynamic interactions occur with medications that alter blood glucose and may require the dose of the oral hypoglycaemic drug to be altered. Pharmacokinetic interactions vary with the drug group. Sulfonylureas and repaglinide are metabolised in the liver. Their plasma concentrations and activity can be reduced by drugs which induce hepatic enzymes and increased by hepatic enzyme inhibitors. Metformin is renally excreted and may have increased toxicity with drugs that impair renal function. Acarbose is only slightly absorbed across the gut and has few significant interactions. Significant interactions with the thiazolidinediones have not yet been reported, but pioglitazone is known to induce cytochrome P450 3A4.

Index words: diabetes, pharmacokinetics, lactic acidosis.

(Aust Prescr 2001;24:83-5)

\section{Introduction}

The sulfonylureas and metformin (a biguanide) have been the mainstay of drug treatment for type 2 diabetes. Recently three new types of drugs have become available; acarbose (an alphaglucosidase inhibitor), repaglinide (a meglitinide) and the 'glitazones' (thiazolidinediones) (Table 1). Drugs from one or more groups are frequently used in combination and have additive effects in lowering blood glucose. The exception to this rule is that repaglinide should not be used with the sulfonylureas since they act through the same final common pathway.

\begin{tabular}{|ll|}
\hline $\begin{array}{l}\text { Table } 1 \\
\text { Oral hypoglycaemic drugs in Australia }\end{array}$ \\
\hline Class & Drug \\
\hline Sulfonylureas & Chlorpropamide \\
& Glibenclamide \\
& Gliclazide \\
& Glimepiride \\
& Glipizide \\
& Tolbutamide \\
Biguanides & Metformin \\
$\alpha$ Glucosidase inhibitors & Acarbose \\
Meglitinides & Repaglinide \\
Thiazolidinediones & Pioglitazone* \\
& Rosiglitazone \\
\hline * Approved but not marketed & \\
\hline
\end{tabular}

All of the oral hypoglycaemic drugs have the potential to interact with other medications and if the result is hypoglycaemia or hyperglycaemia the consequences can be serious. The interactions may be pharmacodynamic (another drug independently raises or lowers blood glucose) or pharmacokinetic (another drug alters the absorption, metabolism or excretion of the hypoglycaemic drug). Both mechanisms may have the effect of changing the apparent efficacy of the hypoglycaemic drugs. Pharmacokinetic interactions may also exacerbate other adverse effects of oral hypoglycaemic drugs.

\section{Pharmacodynamic interactions}

Interactions of this type apply to all classes of hypoglycaemic drugs.

\section{Medications which may raise blood glucose}

Any drug that has the potential to raise blood glucose may produce apparent inefficacy of an oral hypoglycaemic drug. Medications which are commonly reported to increase glucose concentrations are listed in Table 2 with their probable causative mechanisms. In some cases, for example high dose corticosteroids, the patient may need insulin to control their blood glucose until the steroids are ceased.

Stopping a drug which causes hyperglycaemia may produce a significant fall in blood glucose. This may require a parallel reduction in the dose of a hypoglycaemic drug.

\section{Table 2}

Some medications that can raise blood glucose

\section{Drug}

Clonidine

Clozapine

Corticosteroids

Diuretics (especially thiazides)

Nicotinic acid

Nifedipine (but not other calcium antagonists)

Oral contraceptive hormones

Phenytoin

Phenothiazines

Sugar-containing syrups

(e.g. antibiotics/cough mixtures)

\section{Probable mechanism}

Adrenergic action

? Impairs insulin secretion

Oppose insulin action

Oppose insulin action

? Opposes insulin action

Delays insulin action

Oppose insulin action Blocks insulin secretion

Not known

Increased glucose intake
Note: Clinical relevance of some effects is uncertain 


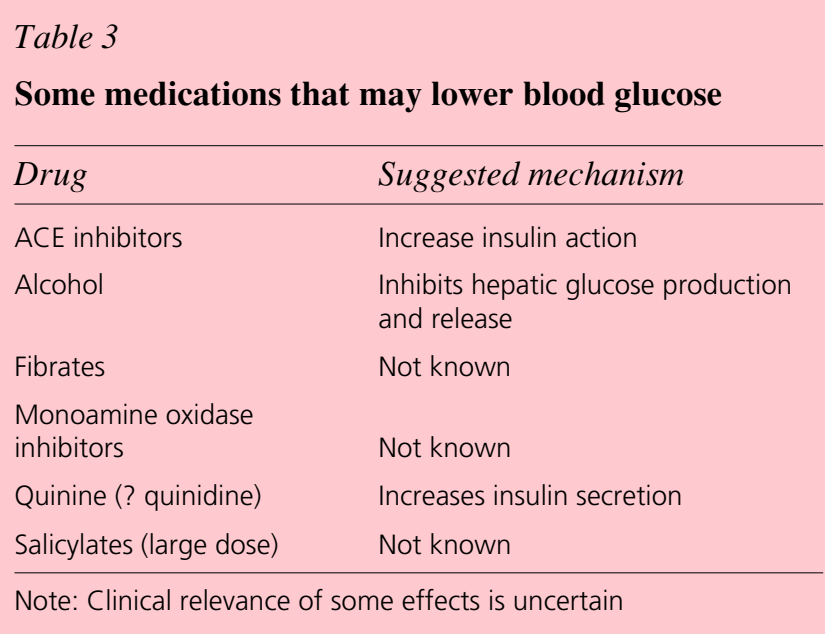

\section{Medications which may lower blood glucose}

Some drugs can lower blood glucose, but the mechanisms of action are not well understood (Table 3). Taking one of these drugs with a hypoglycaemic drug might cause clinically significant hypoglycaemia. The patient may need a lower dose or even have to cease the oral hypoglycaemic drug. Conversely stopping a drug with the potential to lower blood glucose might produce relative inefficacy of a hypoglycaemic drug and create a need for an increased dose.

Beta blockers can mask the warning signs of hypoglycaemia, and the non-selective drugs may impair the normal recovery reaction to hypoglycaemia. There is little evidence that they actually induce hypoglycaemia.

\section{Pharmacokinetic interactions}

These need to be considered separately for each drug class as the body handles the drugs in very different ways and the potential sites for interactions are different.

\section{Sulfonylureas}

All drugs in this class are partially or totally metabolised by the liver. Chlorpropamide is the only member of the class with substantial renal excretion, but is now rarely used. It is excreted much more rapidly in alkaline urine so its half-life and duration of action are reduced with excessive ingestion of alkalis. Antacids may increase the absorption of all the sulfonylureas and hence produce higher peak concentrations of the drugs and a risk of temporary hypoglycaemia.

Chlorpropamide has an additional interaction with alcohol which can produce significant facial flushing. This has been reported with very high doses of tolbutamide, but not with the other drugs in this group.

Sulfonylureas are highly protein bound drugs and may be displaced from blood protein binding sites by drugs such as the non-steroidal anti-inflammatory drugs. This can cause a shortterm increase in free (unbound) sulfonylurea and hence temporary hypoglycaemia.

The majority of significant interactions with sulfonylureas are due to the induction or inhibition of cytochrome P450 enzymes in the liver. Table 4 lists the common interacting drugs and the resultant clinical outcomes. Although dicoumerol interacts

\section{Table 4}

Potential interactions between sulfonylureas or repaglinide and drugs which alter hepatic enzymes

\begin{tabular}{ll}
\hline $\begin{array}{ll}\text { Inducers of metabolism } \\
\text { (reduce concentration of }\end{array}$ & $\begin{array}{l}\text { Inhibitors of metabolism } \\
\text { (increase concentration of }\end{array}$ \\
\hline Phenytoin & hypoglycaemic drug) \\
Phenobarbitone & Allopurinol* \\
Rifabutin & Chloramphenicol \\
Rifampicin & Cimetidine* \\
& Erythromycin \\
* Repaglinide concentrations not increased
\end{tabular}

with tolbutamide it is not used in Australia and there are no significant interactions reported for warfarin or phenindione.

\section{Repaglinide}

This is the only drug of its class currently available in Australia. It has been in use for too short a time for the full spectrum of its potential interactions to have emerged. Like the sulfonylureas it is metabolised by a liver enzyme (cytochrome P450 3A4) and is potentially subject to many of the interactions listed in Table 4.

\section{Metformin}

Metformin is the only biguanide available in Australia. It is not metabolised at all but is completely excreted in urine. Metformin may therefore accumulate and cause lactic acidosis if other medications have induced renal failure. Examples include contrast media, cyclosporin and aminoglycosides. Metformin should therefore be stopped before, and for 48 hours after, contrast radiography.

Metformin is excreted by the renal tubules and this process can be inhibited by cimetidine, but not the other $\mathrm{H}_{2}$ receptor antagonists. This interaction causes retention of metformin and increases the risk of lactic acidosis.

\section{Acarbose}

Acarbose inhibits alpha glucosidase in the gut wall. This reduces the release of glucose from carbohydrate and hence the amount of glucose available for absorption. The drug itself is only absorbed to a minor extent and any interactions relate to interference with its access to the gut lining. In general these interactions would be likely to reduce its efficacy and this has been reported with charcoal and digestive enzyme preparations. In addition neomycin may increase the unpleasant gastrointestinal adverse effects of acarbose.

\section{Thiazolidinediones}

So far three drugs in this group have been marketed but troglitazone has been withdrawn worldwide because of unacceptable hepatotoxicity. Troglitazone also induced cytochrome P450 3A4 and interacted with a number of drugs including cyclosporin and oral contraceptives. So far rosiglitazone and pioglitazone have not shown any significant interactions either in specific studies or in early clinical use. Pioglitazone is known to induce cytochrome P450 3A4 and it 
is possible that interactions with it and with rosiglitazone will become apparent over the next few years.

\section{Conclusion}

Interactions with oral hypoglycaemic agents are important because the outcomes, particularly hypoglycaemia, are serious. As with all interactions the times of high risk are when a second drug is started or stopped or has its dose changed. Regular co-prescription of the same dose of another drug is not likely to cause major problems.

E-mail: gilshen@med.usyd.edu.au

\section{Self-test questions}

The following statements are either true or false

(answers)

1. Drug interactions with metformin are due to induction or inhibition of hepatic enzymes.

2. Patients with diabetes may need to increase the dose of their oral hypoglycaemic drug when they start a course of corticosteroids.

\section{Book review}

Therapeutic Guidelines: Antibiotic. Version 11. North Melbourne: Therapeutic Guidelines Limited; 2000. 317 pages.

Price $\$ 31.90$ (students $\$ 25.30$ ) + \$7.15 postage

Sharon Reid, General Practitioner, Wentworthville, NSW. Lecturer, Department of General Practice, University of Sydney at Westmead Hospital, and Continuing Education Program Manager, Western Sydney Division of General Practice

Therapeutic Guidelines: Antibiotic was first published in 1978. Since the early 1990 s a reasonably up-to-date version of this book has been one of my most used sources of reference when consulting. While my usage of this book was previously limited to a few favourite chapters, the opportunity to review this book has provided the impetus to read it in much more detail. As I expected, given the expertise that has contributed to this book, it appears to be a very useful book for busy clinicians.

Ihave mainly considered the book from the general practitioner viewpoint and have structured my review to address its presentation, organisation and content.

\section{Presentation}

The book is presented in its familiar pink cover and has a wonderful historical 'discovery of antibiotics' graphic on the front. The book seems to be getting a little thicker and the font a little smaller over the years, but in the current version I do not find either feature a major problem. I did wonder if the smaller font size might be problematic for readers with a degree of visual impairment.

\section{Organisation}

The slightly different colours of the various sections of the book facilitate quick access to the content. Future editions would benefit from variation in the colour of the appendices, too.

One other aspect for comment is the listing of drug alternatives. The 'Key information...' section of the book contains the statement 'several drugs are given as alternatives in a list, they are listed alphabetically or in order of preference'. This is confusing, and one or the other scheme should be used throughout.

\section{Content}

Aspects of the book I found particularly useful were:

- the inclusion of statements about the strength of evidence for drug and non-drug therapies

- the introductory chapter 'Principles of antimicrobial use', provides a valuable overview of the basic good practice that will minimise emerging drug resistance

- 'Getting to know your drugs' is a very useful refresher and overview of antimicrobial categories, mechanisms of action, effectiveness and risks

- in the chapters particularly relevant to general practice: (eye, gastrointestinal, genital tract, intra-abdominal, oral and dental, respiratory and skin infections) the topic coverage extends from common minor to more complicated, yet still not uncommon, infections

- the chapter 'Prophylaxis: medical' has a number of useful recommendations including post needle-stick injury prophylaxis

- 'Antimicrobials and food' answers questions patients often ask about

- the appendices 'Pregnancy and breastfeeding' and 'Paediatric doses' are both useful quick references.

While a few of the chapters and appendices are less useful for day-to-day general practice, they are helpful for keeping up to date with inpatient treatments and as a source of information for general practitioners caring for patients in hospital.

Overall I found Version 11 to be a practical, concise and informative book. Despite a few minor imperfections in the organisation and presentation of the book I would recommend it highly to the busy general practitioner. In addition, because of its breadth of cover and summarised format, it is likely to be of value to specialist clinicians outside their area of expertise, clinicians in training and medical students. 